Engineering and Design

Elsevier Editorial System(tm) for Fusion

Manuscript Draft

Manuscript Number:

Title: ITER ECRH Upper Launcher: Test plan for qualification of the Diamond Torus Window Prototype III

Article Type: SI:ISFNT-12

Keywords: ITER, ECRH Upper Launcher (ECHUL), Diamond window, CVD, prototype

Corresponding Author: Dr. Sabine Schreck,

Corresponding Author's Institution: Karlsruhe Institute of Technology

First Author: Sabine Schreck

Order of Authors: Sabine Schreck; Gaetano Aiello; Andreas Meier; Dirk Strauss; Mario Gagliardi; Gabriella Saibene; Theo Scherer

Abstract: The diamond window is part of the electron cyclotron heating upper launcher system for ITER. Together with the isolation valve it constitutes the primary vacuum boundary and it also acts as first tritium barrier. Therefore the window is classified as Safety/Protection Important Component (SIC/PIC) with the nuclear safety function "confinement". As the diamond window unit is not entirely covered by standard codes, an ad-hoc qualification program needs to be defined, including analysis, prototyping and testing. In the framework of a contract with F4E, the test program for a diamond window prototype is being developed with the aim to prove its operability for normal, accidental and incidental conditions as identified in the ITER load specifications. Tests range from dielectric loss measurements for the bare Chemical Vapour Deposition (CVD) diamond disk up to mechanical and vacuum tests for the complete window assembly. Finally mm-wave properties have to be characterized for the complete window. A clear definition of the testing requirements and of the acceptance criteria is necessary as well as a complete documentation of the process.

This paper will present the development of the test plan for a window prototype, which is currently under manufacturing. First tests are directed to the characterisation of the bare diamond disk with a focus on its dielectric properties. 


\section{ITER ECRH Upper Launcher: \\ Test plan for qualification of the Diamond Torus Window Prototype III}

Sabine Schreck ${ }^{\mathrm{a}}$, Gaetano Aiello ${ }^{\mathrm{a}}$, Andreas Meier ${ }^{\mathrm{a}}$, Dirk Strauss ${ }^{\mathrm{a}}$, Mario Gagliardi ${ }^{\mathrm{b}}$, Gabriella Saibene ${ }^{\mathrm{b}}$ and Theo Scherer $^{\mathrm{a}}$

${ }^{a}$ Karlsruhe Institute of Technology, Institute for Applied Materials, Hermann-von-Helmholtz-Platz 1,

76344 Eggenstein-Leopoldshafen, Germany

${ }^{b}$ F4E, Antennas and Plasma Engineering, Josep Pla 2, Torres Diagonal Litoral B3,

08019 Barcelona, Spain 
Highlights:

An ad- hoc qualification program for the ITER EC H\&CD Launcher diamond torus window is being developed in a framework contract between KIT and F4E.

The testing program for the qualification of the bare diamond disk is defined.

Diamond disk prototypes have been manufactured and the qualification tests of the disks are under execution. First results show a very good quality of the diamond disks. 


\title{
ITER ECRH Upper Launcher: Test plan for qualification of the Diamond Torus Window Prototype III
}

\author{
Sabine Schreck ${ }^{\mathrm{a}}$, Gaetano Aiello ${ }^{\mathrm{a}}$, Andreas Meier ${ }^{\mathrm{a}}$, Dirk Strauss ${ }^{\mathrm{a}}$, Mario Gagliardi $^{\mathrm{b}}$, Gabriella Saibene $^{\mathrm{b}}$ and Theo Scherer $^{\mathrm{a}}$ \\ ${ }^{a}$ Karlsruhe Institute of Technology, Institute for Applied Materials, Hermann-von-Helmholtz-Platz 1, \\ 76344 Eggenstein-Leopoldshafen, Germany
}

${ }^{b}$ F4E, Antennas and Plasma Engineering, Josep Pla 2, Torres Diagonal Litoral B3, 08019 Barcelona, Spain

The diamond window is part of the electron cyclotron heating upper launcher system for ITER. Together with the isolation valve it constitutes the primary vacuum boundary and it also acts as first tritium barrier. Therefore the window is classified as Safety/Protection Important Component (SIC/PIC) with the nuclear safety function "confinement". As the diamond window unit is not entirely covered by standard codes, an ad-hoc qualification program needs to be defined, including analysis, prototyping and testing. In the framework of a contract with F4E, the test program for a diamond window prototype is being developed with the aim to prove its operability for normal, accidental and incidental conditions as identified in the ITER load specifications. Tests range from dielectric loss measurements for the bare Chemical Vapour Deposition (CVD) diamond disk up to mechanical and vacuum tests for the complete window assembly. Finally mm-wave properties have to be characterized for the complete window. A clear definition of the testing requirements and of the acceptance criteria is necessary as well as a complete documentation of the process.

This paper will present the development of the test plan for a window prototype, which is currently under manufacturing. First tests are directed to the characterisation of the bare diamond disk with a focus on its dielectric properties.

Keywords: ITER, ECRH Upper Launcher (ECHUL), Diamond window, CVD, prototype

\section{Introduction}

The ITER ECRH system consists of one equatorial EC launcher (EL) and four upper launchers (UL). The upper launchers are connected via transmission lines to the ITER gyrotrons providing up to $20 \mathrm{MW}$ mm-wave power at $170 \mathrm{GHz}$ and the main purpose is to drive local current with the aim to control the plasma instabilities (mitigate NTMs) [1]. The diamond window is located in the port cell environment between the launcher and the transmission line (Fig. 1). The diamond window unit consists of a synthetic polycrystalline diamond disk produced by 'Microwave Plasma Assisted (MPA)'

chemical vapour deposition (CVD) mounted in a system of metallic parts (copper/steel). The requirements to such a CVD diamond window are an ultra-low loss tangent of the disk $\left(\tan \delta<2 \times 10^{-5}\right)$ and a robust structural design against the expected loads allowing the transmission of up to $1.5 \mathrm{MW}$ into the plasma via the launcher.

The window unit is part of the first ITER confinement system and thus it has the most stringent requirements in the ITER safety (SIC1/PIC1), quality, vacuum, seismic and tritium classifications.

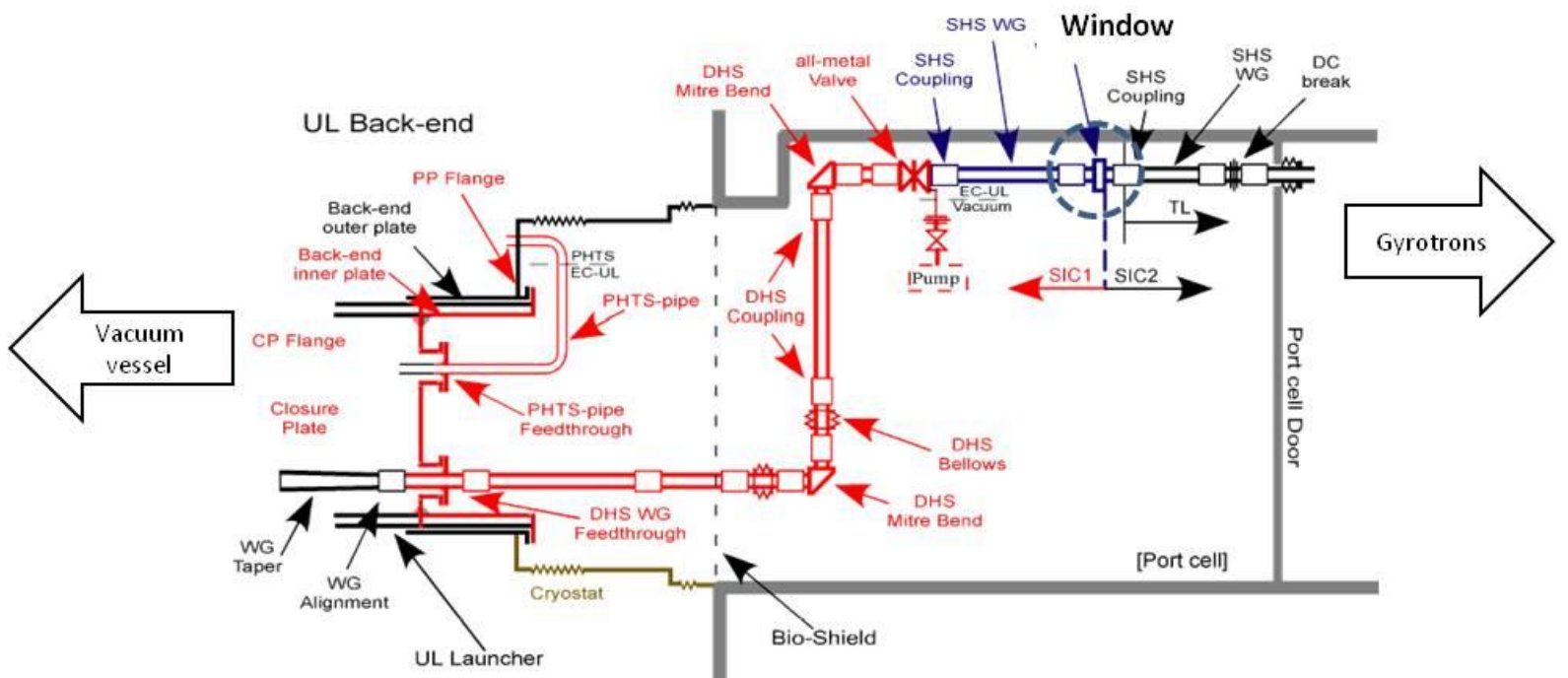

Fig. 1. A schematic (from EC Safety System Philosophy-ITER_D_BN7UPS) illustrating the principle waveguide components in the port cell region. The diamond window is a SIC-1 component located directly at the barrier to SIC 2. 
As the design, the manufacturing and the qualification of the diamond window cannot be completely covered by standard codes, a specific qualification program has to be developed. Within this program, acceptance criteria for clearly defined tests have to be determined in order to guarantee the overall functionality of the component. The testing and qualification plan needs to respect and fulfil the requirements of nuclear safety, as postulated in the ITER Preliminary Safety Report (RPrS). All testing conditions have to be compliant with the design driver loading conditions expected during the lifetime of an ITER window. A definition of damage limits is necessary and the test results have to be compared against these limits. The complete procedure needs to be accompanied by supporting analysis.

In the framework of a contract with F4E, KIT develops the testing and qualification program for a window prototype and will execute the prototype tests. The tests range from standard tests for geometry control, vacuum and leakage tests up to mm-wave-transmission tests for which a dedicated measurement facility (FABRY-PEROT-resonator) is available at KIT.

The prototype to be tested (also called Prototype III) is built based on the design proposed by KIT, which is expected to represent the final design of the ITER ECRH UL window. Two disks procured by F4E to be used for the window prototype were already manufactured by the company Diamond Materials (Freiburg, Germany) and first results on their dielectric properties are available.

\section{Updated window design for Prototype III}

With respect to the previous window prototypes (Prototype I and Prototype II) [2], the design of the window was updated and optimized by FEM analyses and the application of the ASME Sec. III - Subsection NC code. This is the selected code for the design, manufacturing, assembling (i.e. the joining) and qualification of the diamond window unit, being classified as an ASME class 2 component. The design driver load combination for the unit was taken into account in the FEM analyses [3]. The updated window design is shown in Fig. 2 and Fig. 3. The window unit basically consists of a $1.11 \mathrm{~mm}$ thick (Ø $80 \mathrm{~mm})$ diamond disk brazed to two oxygen-free copper cuffs and this structure is then integrated into a metallic housing by welding. Two nickel rings, named spacer rings, connect the cuffs to corrugated stainless steel waveguides, which are inserted into the cuffs leaving a $100 \mu \mathrm{m}$ gap with the diamond disk. This $100 \mu \mathrm{m}$ have been evaluated during Prototype II manufacturing and testing (within F4E-OPE140) [2, 4].

In general, the design strategy was to have a very rigid outer window frame able to withstand the external loads acting on the unit while thin copper cuffs brazed to the diamond allow indirect cooling of the disk (i.e., no direct contact between disk and coolant). Diagnostic pipes have been added to allow real-time monitoring of the interspaces inside the unit. The brazing is carried out only between the disk and the cuffs while all other parts are joined by welding.
The requirements for the manufacturing, assembling and examination of the window unit prototype according to ASME and according to the mm-wave properties of the window are collected in a Technical Specification (TS) document. This also provides the specifications for the testing at manufacturer site of pre-manufacturing mock-ups and of the diamond window assembly itself. One general requirement is to produce an appropriate documentation for the manufacturing process including equipment descriptions, process parameters, environmental conditions, material certificates, etc.

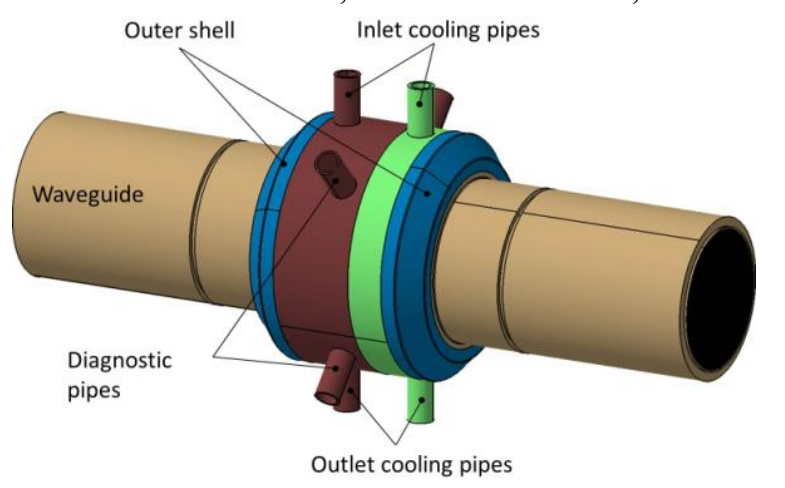

Fig. 2. Current design of the ITER window unit.

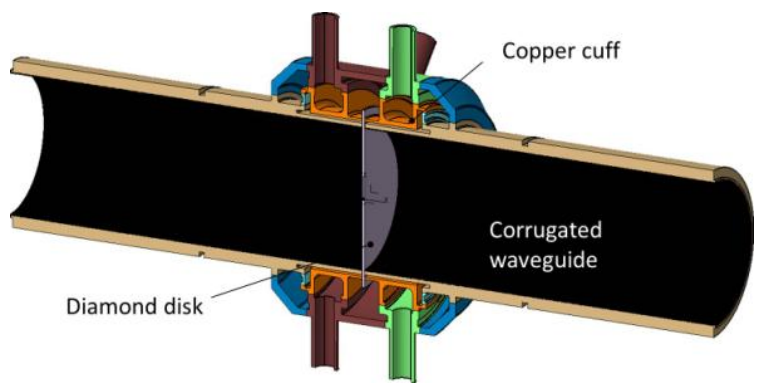

Fig. 3. Section view of the ITER window unit.

\section{Prototype test and qualification program}

The testing program for the window assembly can be split into different parts. Selected tests are executed by the window manufacturer. Other tests have to be performed by qualified labs, e.g. the dielectric properties measurements. Particular tests are required for the bare disk, for the disk already brazed to the copper cuffs and for the complete assembly.

As for the manufacturing process also all tests have to be supplemented by an extensive documentation, including description of test procedures, data acquisition plans, calibration of the measurement setups, certifications of used materials, error and uncertainty analysis etc.. This includes also specific Control Plans for selected procedures.

\subsection{Qualification of bare diamond disk}

Fig. 4 shows the main steps of the disk qualification. After the disk has passed the internal manufacturer characterization, it is transported to KIT following the rules for transport and logistic. Only if the diamond disk complies with all specifications, the shipping to the window assembler company will be carried out. Table 1 gives an excerpt of the acceptance criteria. 


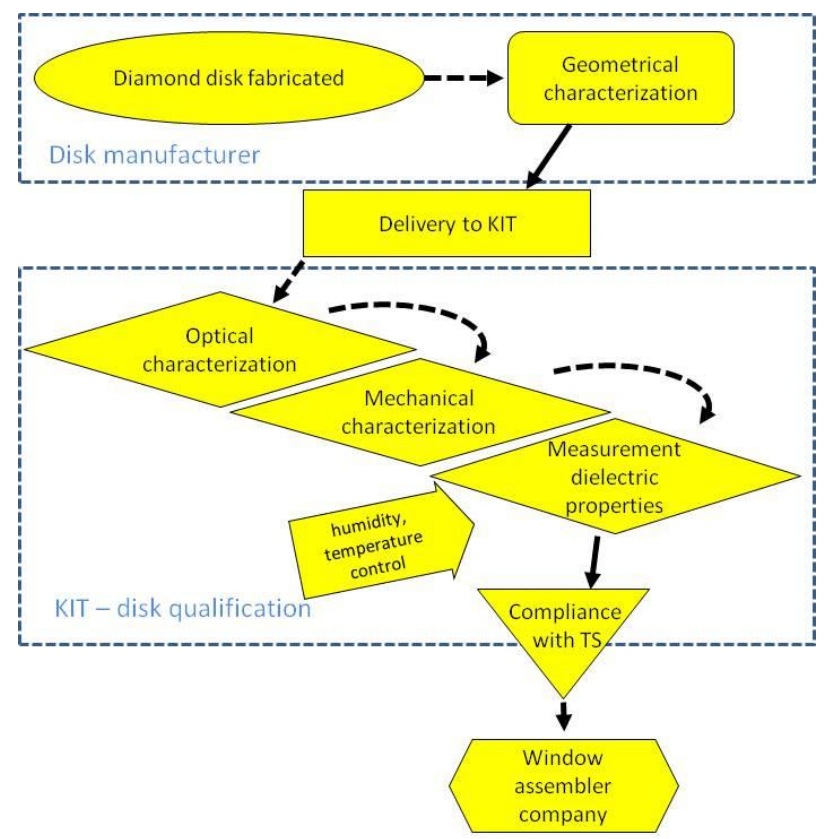

Fig. 4. Main steps for disk qualification.

Table 1. Excerpt of acceptance criteria from the Technical Specifications (TS) of the diamond disk.

\begin{tabular}{ll}
\hline Parameter & Value (with tolerances) \\
\hline diameter & $80 \mathrm{~mm}(+0.2 /-0.2 \mathrm{~mm})$ \\
thickness & $1.11 \mathrm{~mm}$ \\
& $(+0.005 \mathrm{~mm} /-0.000 \mathrm{~mm})$ \\
Planarity (flatness) of disk & $10 \mu \mathrm{m}$ \\
$\begin{array}{l}\text { Surface quality: } \\
\text { Ra roughness (Ra) }\end{array}$ & $20 \mathrm{~nm}$ \\
$\begin{array}{l}\text { loss tan } \delta @ 170 \mathrm{GHz} \\
\text { (mean D50) }\end{array}$ & $<3.5 \cdot 10^{-5}\left(+5 \cdot 10^{-6}\right)$ \\
$\begin{array}{l}\text { loss tan } \delta @ 170 \mathrm{GHz} \\
\text { (mean D90) }\end{array}$ & $<6.0 \cdot 10^{-5}\left(+1 \cdot 10^{-5}\right)$ \\
\hline
\end{tabular}

\section{A) Geometrical characterization}

After fabrication via the CVD process, the required disk thickness and surface quality has to be adjusted by grinding. Several steps for geometrical characterization are necessary to verify the compliance with the TS for the disk. The surface roughness $\mathrm{Ra}$ and thickness distribution is measured by tactile or optical methods at the manufacturer side. The planarity is be measured by interferometry (fringe measurement) using FIZEAU or TWYMAN-GREEN setups [5]. All test results have to be reported in a manufacturing report (e.g., thickness (central, max. and min.), diameter, roughness and planarity).

\section{B) Optical characterization}

After delivery of the disk to KIT, the standard procedure to detect large visible cracks is to perform an optical scanning of the diamond disk (24 bit color depth, min. resolution $500 \mathrm{dpi}$ ). In the case of occurrence of visible black structures in the central disk area with a diameter of $63.5 \mathrm{~mm}$ (free aperture of the microwave beam due to the waveguide inner diameter), a RAMAN measurement will be performed. The RAMAN-setup is able to distinguish between diamond like carbon (DLC), graphite and pure diamond phases in the disk. Graphite like phases or other impurities will increase the loss tangent of the disk and have to be therefore minimized.

\section{C) Mechanical characterization (bow under pressure)}

For the characterization of the mechanical properties, a measurement of the disk bow (from both sides) under standard pressure $(\mathrm{p}=1013 \mathrm{mbar})$ and vacuum $(p<100$ mbar) is carried out. According to current stress calculations for a $1.11 \mathrm{~mm}$ disk, the maximum limit for the bow is $70 \mu \mathrm{m}$ considering brazing effect and 1 bar pressure.

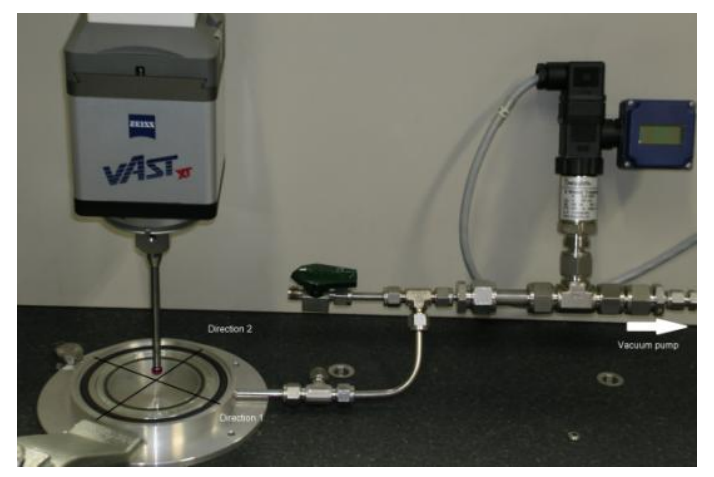

Fig. 5: Pressure dependent bow measurement of a diamond disk at KIT (ZEISS Contura; typically, a tactile measurement with 15 points in horizontal and vertical direction is sufficient to achieve the required accuracy.)

\section{D) Dielectric measurements on the pure CVD diamond disk}

For the quality control of the microwave properties of a diamond disk, a dedicated measurement facility with different FABRY-PEROT-Resonators (spherical and hemi-spherical) is available at KIT. The loss qualification is made by open resonator setups which consist of a spherical mirror and a plane mirror or two spherical mirrors.

Taking into account the measured resonant frequency and quality factor (ratio of the mean stored energy and the dissipated energy per period of the oscillation) of the empty unloaded resonator $\left(f_{0}, Q_{0}\right)$, and the same for the loaded resonator $\left(f_{m}, Q_{m}\right)$, the dissipative part of the complex permittivity $\left(\varepsilon_{\mathrm{r}}{ }^{*}=\varepsilon_{\mathrm{r}}{ }^{\prime}+\mathrm{i} \cdot \varepsilon_{\mathrm{r}}{ }^{\prime \prime}\right)$ can be determined. The ratio of the dissipative imaginary and real part of the permittivity is the loss tangent $\tan \delta$ and is the characteristic quantity for the qualification.

The hemi-spherical resonator (= mapping setup) is composed of a spherical mirror with coupling holes to form the electromagnetic wave and a reflecting plane mirror (see Fig. 6a). The formed resonance is determined relating to frequency $\left(\mathrm{f}_{0}-\right.$ empty, $\mathrm{f}_{\mathrm{m}}$-filled) and quality factor $\left(Q_{0}, Q_{m}\right)$ (Fig. 6b) [6]. With the hemi-spherical setup the distribution of the dielectric loss over the diamond disk can be measured and is parameterized in terms of the onset (D10), median (D50) and terminal (D90) distribution parameters. These indicate the loss tangent for $10 \%, 50 \%$ and $90 \%$ fraction of the inspected area. As an example, a typical result of a disk mapping is shown in Fig. 7. The loss tangent mapping is only possible for bare diamond disks. 
a)

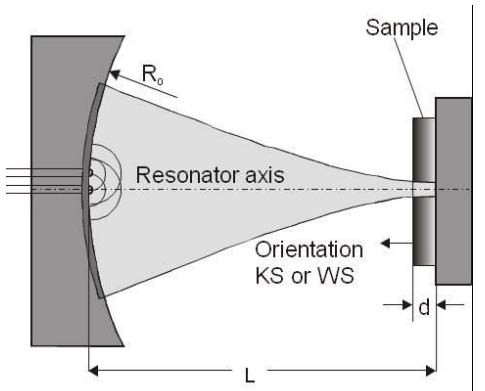

b)

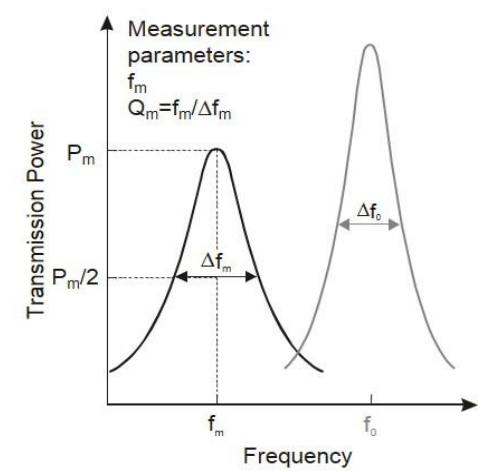

Fig. 6. a) Schematics of the hemi-spherical setup [6] and b) measurement parameters for the empty and filled resonator.

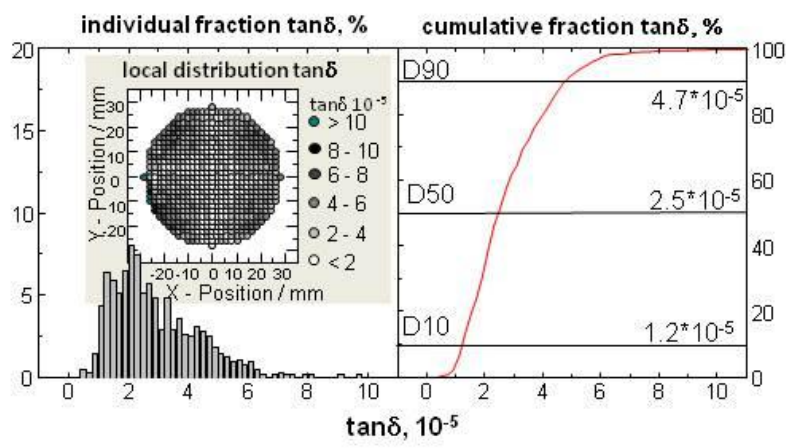

Fig. 7. Dielectric loss mapping of a typical bare CVD diamond window disk (@ f=145 GHz).

High resolution measurements for the central $\tan \delta$ of the disk are possible with a symmetrical resonator setup. This is formed by two spherical mirrors and the specimen is placed at the center (Fig. 8 a, b) [7]. The microwave is weakly coupled in and out of the resonator by using a $45^{\circ}$ oriented Polytetrafluoroethylene (PTFE) foil. The sample is axially (x) shifted by a step motor through the electromagnetic field and the resonator parameters loaded quality factor $\mathrm{Q}_{\mathrm{m}}$ and central frequency $f_{m}$ are measured. A comparison with the values of the empty resonator allows the calculation of $\tan \delta$. The theory can be found in [7].

The dielectric measurement for the disk-cuff subassembly is only possible with this spherical resonator arrangement.

To conclude, the following measurements are necessary for the dielectric characterization of the disk at $170 \mathrm{GHz}$ :

1. Measurement of the permittivity $\varepsilon_{\mathrm{r}}$ and the loss tangent $\tan \delta$ in transmission (hemi-spherical resonator).

2. Mapping of the whole disk - determination of D10, D50 and D90 (hemi-spherical resonator).
3. Measurement of $\tan \delta$ in resonant mode (spherical resonator) at the centre of the disk.

4. In case of pollutions: chemical cleaning procedure of the diamond surfaces and re-do steps 1) - 3).

Each measurement has to be carried out together with a reference sample measurement under the same conditions (temperature, humidity).

Disks with maximum loss tan $\delta @ 170 \mathrm{GHz}$ (mean D50) of $3.5 \cdot 10^{-5}$ and (mean D90) of $6.0 \cdot 10^{-5}$ are acceptable to be integrated into the window assembly. This assures low power dissipation in the disk and therefore a very low heating of the window unit [8].

a)

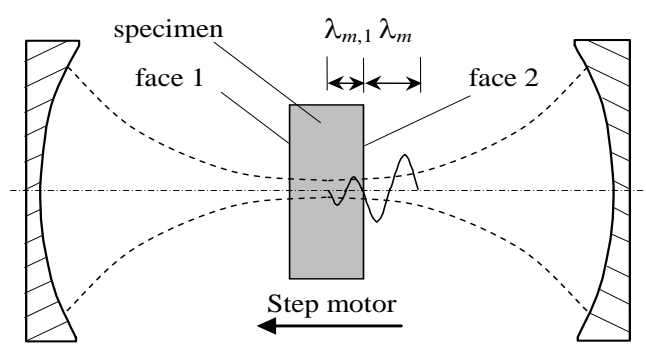

b)

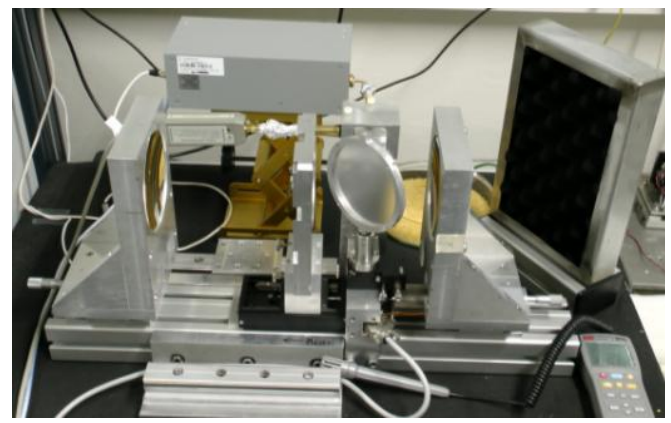

Fig. 8. a) Scheme of the loaded spherical resonator and b) Photograph of it.

\subsection{Qualification of window sub-assembly}

The brazing of the disk to the cupper cuff is an important manufacturing step. Various qualification tests of this sub-assembly (diamond disk, copper cuffs, cooling rings) are necessary before the complete window assembly can proceed. First of all the leak tightness (between cuff and disk = brazing seam) has to be verified by a helium leak test (manufacturer site). The concentricity of cuffs of both sides (alignment) and the tolerances is controlled by using a Coordinate Measurement Machine (CMM). In a next step, an optical inspection has to follow and has to be completed by a detailed inspection of the brazing seam itself, by the mean of X ray-Computer tomography. KIT can provide a high-resolution system for 2D X-ray inspection and 3D computed tomography (phoenix $v \mid$ tome $\mid \mathrm{x} s$ ) for the purpose to identify voids, their size and distribution inside the brazing seam.

Using a white light profilometer the bowing of the disk inside the sub-assembled housing is checked. Finally the central dielectric loss is re-measured after the disk is joined to the copper cuffs to exclude possible pollution on the disk surface originated from the brazing process. If all acceptance criteria are fulfilled the sub-assembly will be delivered to the manufacturer for final assembly. 


\subsection{Qualification of the complete window unit}

The main steps for qualification of the completed window prototype are shortly summarized here:

a) Geometry control of the window unit by CMM.

b) Water flow and pressure tests $(<10$ bar) of the cooling circuit. (The window will be connected to the CCWS 1 of the ITER system)

c) Vacuum test (both sides and disk interspace)

d) Tests of the high power RF performance: short pulse experiments $(\tau=3-5 \mathrm{sec})$ with power up to $\mathrm{P}=0.5 \mathrm{MW}$ and long pulse experiments (up to $500 \mathrm{sec}$ ) up to $1 \mathrm{MW}$.

\section{Prototype Testing}

The various tests for the window prototype are performed by the disk and/or window manufacturer and by KIT. Currently, two diamond disks are available for their integration into the window assembly. Some tests have been already performed and the results are summarized in the following paragraph.

\subsection{Testing of the bare diamond $\operatorname{disk}(\mathrm{s})$}

A geometrical characterization has been made by the disk manufacturer (Diamond Materials Freiburg) and the disk complies with the requirements. In Fig. 8 a photo of one of the disks is shown (T02-DM). At the lower edge the laser engraving is visible. This is necessary for ensuring its unique identification. The disk is transparent and shows no significant inclusions.

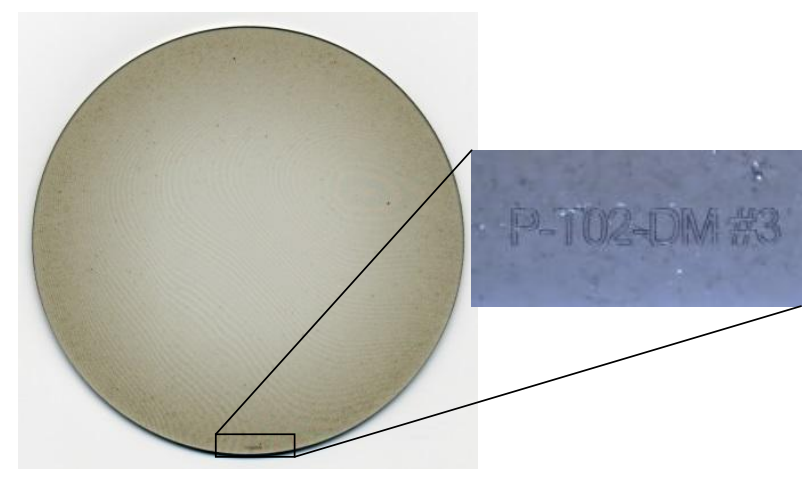

Fig. 9. Photo of disk T02-DM

The central loss tangent measurements made with the spherical resonator setup (Fig. 8) are shown together with some geometrical properties in Table 2.

Table 2. Excerpt of disk properties

\begin{tabular}{lll}
\hline Parameter & T02-DM & T03-DM \\
\hline diameter & $79.95 \mathrm{~mm}$ & $79.96 \mathrm{~mm}$ \\
thickness (central) & $1.1149 \mathrm{~mm}$ & $1.1148 \mathrm{~mm}$ \\
planarity & & \\
nucleation side & $0.52 \mu \mathrm{m}$ & $0.83 \mu \mathrm{m}$ \\
growth side & $0.66 \mu \mathrm{m}$ & $1.43 \mu \mathrm{m}$ \\
Ra roughness & & \\
nucleation side & $3.86 \mathrm{~nm}$ & $4.13 \mathrm{~nm}$ \\
growth side & $2.11 \mathrm{~nm}$ & $3.91 \mathrm{~nm}$ \\
central loss tan $\delta$ & $(3.6 \pm 0.9) \cdot 10^{-6}$ & $(4.2 \pm 1.1) \cdot 10^{-6}$ \\
& $169.57 \mathrm{GHz}$ & $169.58 \mathrm{GHz}$ \\
permittivity $\varepsilon_{\mathrm{r}}$ & 5.67 & 5.67 \\
\hline
\end{tabular}

The low values of the central loss tangent indicate a very high quality of the disks and KIT's measurement set-up runs at its resolution limit. The mapping, which will give the important information on the $\tan \delta$ distribution, still needs to be completed.

\section{Summary and Conclusions}

Within the running contract between F4E and KIT, the technical specifications for the prototypes of the diamond disk and the window unit have been defined. The testing and qualification program of the bare disk(s) is specified and under execution showing a high quality of the available diamond disks. The test program of the window assembly is under definition. During the test phase for the window prototype III all specifications will be reviewed and possibly tuned to the needs and to potential floating requirements.

The definition of the tests, their first-time execution and documentation based on the window prototype are important steps in advance to the procurement.

\section{Acknowledgments}

This work was supported by Fusion for Energy under the service contract No F4E-OPE-467. The views and opinions expressed herein reflect only the author's views. Fusion for Energy is not liable for any use that may be made of the information contained therein.

\section{References}

[1] D. Strauss, G. Aiello, A. Bruschi, R. Chavan, D. Farina, L Figini, Progress of the ECRH upper launcher design for ITER, Fusion Eng. Des. 89, 2014, 1669-1673.

[2] Article in press: S. Schreck, et al., ITER ECRH upper launcher torus diamond window - Prototyping, testing and qualification, Fusion Eng. Des. (2015), http://dx.doi.org/10.1016/j.fusengdes.2015.02.002

[3] G. Aiello et al., The ITER EC H\&CD Upper Launcher: Methodology in the FEM Analyses of the Diamond Window Unit Subject to Seismic and Baking Loads, Proceedings of the $26^{\text {th }}$ IEEE SOFE conference, Austin, Texas USA, 2015.

[4] T. A. Scherer et al., Recent upgrades of the ITER ECRH CVD torus diamond window design and investigations of dielectric diamond properties, In: Electron Cyclotron Emission and Electron Cyclotron Resonance Heating (EC-16), 2011, 396-400.

[5] M. Vannoni, G. Molesini, Three-flat test plates in horizontal posture, Appl. Optics, Vol.47, No. 12 (2008), pp. 2133-2145

[6] F. Königer: Measurement system for the precise determination of dielectrical properties in the mm-wave range based on hemispherical open resonators. Frequenz 43 (1989)

[7] I. Danilov, R. Heidinger, New approach for open resonator analysis for dielectric measurements at $\mathrm{mm}$ wavelengths, Journal of the European Ceramic Society 23 (2003)

[8] T. A. Scherer et al., Experimental analysis of the inserted waveguide CVD diamond window prototype for the ITER ECRH upper launcher, In: Infrared, Millimeter and Terahertz Waves (IRMMW-THz), 2011. 